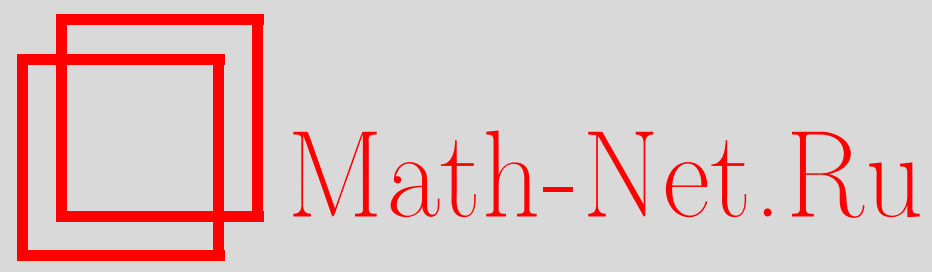

Д. С. Ткаченко, Об одной обратной задаче для параболического уравнения, Матем. заметки, 2004, том 75, выпуск 5, 729-743

DOI: https://doi.org/10.4213/mzm71

Использование Общероссийского математического портала Math-Net.Ru подразумевает, что вы прочитали и согласны с пользовательским соглашением http://www.mathnet.ru/rus/agreement

Параметры загрузки:

IP : 54.80 .97 .219

26 апреля 2023 г., $17: 44: 25$ 


\section{ОБ ОДНОЙ ОБРАТНОЙ ЗАДАЧЕ \\ ДЛЯ ПАРАБОЛИЧЕСКОГО УРАВНЕНИЯ}

\section{Д. С. Ткаченко}

В работе исследуется обратная задача восстановления правой части специального вида для параболического уравнения по $u$ с зависящими от $(x, t)$ коэффициентами перед $u_{t}$ и $u$. При этом в качестве переопределения задается интеграл от решения по времени. Доказана фредгольмовость такой задачи, теорема существования и единственности ее решения в соболевских пространствах.

Библиографоия: 14 названий.

\section{1. Постановка задачи. Формулировка результатов}

1.1. Постановка задачи. Пусть $\Omega$ - ограниченная, односвязная область в $\mathbb{R}^{n} \mathrm{c}$ границей $\partial \Omega \in C^{2}$. В цилиндре $Q=\Omega \times(0, T)$, где $T>0$, с боковой поверхностью $S=\partial \Omega \times(0, T)$ рассматривается начально-краевая задача для параболического уравнения. При этом неизвестной предполагается не только “температура", но и не зависящая от времени компонента "источника", а в качестве дополнительной информации, или “переопределения", задается интеграл “температуры" по отрезку [0,T]. Подобные задачи в различных пространствах исследовались рядом авторов [1]-[8]. Однако в большинстве работ рассматривался стационарньй оператор левой части уравнения. В работе [3] в одномерном случае удалось отказаться от стационарности оператора, но обратная задача (с финальным переопределением) рассматривалась в гельдеровых пространствах. Мы приводим подробное доказательство существования единственного решения обратной задачи “в малом" в соболевских пространствах без ограничений на размерность. При этом коэффициенты перед $u_{t}$ и $u$ предполагаются зависящими от $(x, t)$. В качестве вспомогательного результата приводится оценка некоторых норм решения прямой задачи для случая, когда все коэффициенты зависят от $(x, t)$.

В работе рассматривается следующая обратная задача.

ЗАДАчА 1. Найти пару $u \in W_{2,0}^{2,1}(Q), p \in L_{2}(\Omega)$ из условий

$$
\begin{aligned}
\rho(x, t) u_{1} t-L(x, t) u_{1} & =f_{1}(x, t)=\Phi_{1}(x, t) p(x)+q(x, t), & (x, t) & \in Q, \\
\left.u_{1}\right|_{t=0} & =u_{0}(x), & x & \in \Omega,
\end{aligned}
$$

Работа выполнена при поддержке Российского фонда фундаментальных исследований, грант № 00-01-00638. 


$$
\begin{array}{rrr}
\left.u_{1}\right|_{S}=0, & (x, t) \in S, \\
l_{1} u_{1}=\int_{0}^{T} \omega_{1}(t) u_{1}(x, t) d t=\chi_{1}(x), & x \in \Omega,
\end{array}
$$

әде оператор $L u=\sum_{i, j=1}^{n}\left(a_{i, j}(x) u_{x_{i}}\right)_{x_{j}}-\sum_{i=1}^{n} b_{i}(x) u_{x_{i}}-c(x, t) u$ равномерно әллиптичный с достаточно гладкими коэффициентами (точные условия на них даны ниже), а все остальнье, входящие в (1.1)-(1.4) функиии, определень и принадлежат следующим классам:

$$
\Phi_{1}, \Phi_{1 t} \in L_{\infty}(Q), \quad q, q_{t} \in L_{2}(Q), \quad u_{0}, \chi_{1} \in W_{2,0}^{2}(\Omega), \quad \omega_{1} \in L_{2}(0, T) .
$$

Пространства и нормы, используемые в работе, вводятся общепринято; в частности, нормы в $L_{2}(\Omega), W_{2}^{1}(\Omega), W_{2}^{2}(\Omega)$ и $W_{2}^{2,1}(Q)$ определяются, соответственно, равенствами

$$
\begin{gathered}
\|v\|_{2, \Omega}=\left(\int_{\Omega} v^{2}(x) d x\right)^{1 / 2}, \quad\|v\|_{2, \Omega}^{(1)}=\left(\int_{\Omega}\left(v^{2}(x)+v_{x}^{2}(x)\right) d x\right)^{1 / 2}, \\
\|v\|_{2, \Omega}^{(2)}=\left(\int_{\Omega}\left(v^{2}(x)+v_{x}^{2}(x)+v_{x x}^{2}(x)\right) d x\right)^{1 / 2}, \\
\|u\|_{2, Q}^{(2,1)}=\left(\int_{Q}\left(u^{2}+u_{t}^{2}+u_{x}^{2}+u_{x x}^{2}\right) d x d t\right)^{1 / 2}
\end{gathered}
$$

где $u_{x}^{2}=\sum_{i=1}^{n} u_{x_{i}}^{2}, u_{x x}^{2}=\sum_{i, j=1}^{n} u_{x_{i} x_{j}}^{2}$, пространство $W_{2,0}^{2,1}(Q)=\left\{u \in W_{2}^{2,1}(Q)\right.$ : $\left.\left.u\right|_{S}=0\right\}$. Также нам понадобится пространство $L_{\infty}(\Omega)$ с нормой

$$
\|v\|_{\infty, \Omega}=\underset{\Omega}{\operatorname{ess} \sup }|v(x)| \equiv \inf \{M \in \mathbb{R}:|v| \leqslant M \text { для п.в. } x \in \Omega\},
$$

энергетическое пространство $V_{2}(Q)$ с нормой

$$
\|v\|=\operatorname{essup}_{[0, T]}\|v\|_{2, \Omega}+\left\|v_{x}\right\|_{2, Q}
$$

и $V_{2}^{1,0}(Q)$, состоящее из тех элементов $V_{2}(Q)$, для которых при каждом $t \in[0, T]$ существует след $u(x, t) \in L_{2}(\Omega)$, причем $\|u(x, t+\Delta t)-u(x, t)\|_{2, \Omega} \rightarrow 0$ при $t \rightarrow 0$.

В обозначениях $W_{2}^{1}(\Omega)$ и $\stackrel{\circ}{2}_{2}^{1,0}(Q)$ нуль сверху означает равенство нулю следов элементов данных пространств на границе $\partial \Omega$ и $S$ соответственно. Все эти пространства подробно описаны, например, в [9, гл. $1, \S 1]$ или $[1$, гл. 1, п. 1.1].

Все равенства и неравенства (кроме числовьх и тех, смысл которых поясняется отдельно), встречающиеся в тексте, понимаются в смысле “почти всюду" по мере Лебега.

ОПРЕДЕЛЕНИЕ 1.1. Пара функций $u \in W_{2,0}^{2,1}(Q), p \in L_{2}(\Omega)$, называется решением задачи (1.1)-(1.4), если выполняются равенства (1.1)-(1.4).

Всюду далее будем предполагать выполненными следующие условия:

$$
\begin{gathered}
\rho(x, t) \in C^{2}(\bar{Q}), \quad 0<\rho_{0} \leqslant \rho(x, t) \leqslant \rho_{1}, \quad \partial \Omega \in C^{2}, \\
a_{i j} \equiv a_{j i}(x) \in C^{2}(\bar{\Omega}), \quad b_{i}(x) \in C^{1}(\bar{\Omega}), \quad c(x, t) \in C^{1}(\bar{Q}),
\end{gathered}
$$


существуют константы $\nu_{1}, \nu_{2}: 0<\nu_{1}<\nu_{2}$,

$$
\nu_{1}|\xi|^{2} \leqslant a_{i j} \xi_{i} \xi_{j} \leqslant \nu_{2}|\xi|^{2}, \quad x \in(\bar{\Omega})
$$

(здесь и далее всюду, где это не может привести к путанице, знаки суммы по всем возможным значениям индексов опускаются),

$$
\begin{gathered}
\Phi_{1}, \Phi_{1 t} \in L_{\infty}(Q), \quad g(x)^{-1} \equiv\left(l_{1}\left(\Phi_{1}\right)\right)^{-1} \in L_{\infty}(\Omega), \\
0 \not \equiv \omega L_{2}(0, T), \quad \chi(x) \in W_{2,0}^{2}(\Omega) .
\end{gathered}
$$

\section{2. Основные результаты. Введем обозначения}

$$
\Phi(x, t)=\frac{\Phi_{1}(x, t) e^{-\lambda t}}{g(x)}, \quad \varphi(x)=p(x) \cdot g(x), \quad \text { где } g(x) \equiv l_{1}\left(\Phi_{1}\right) ; \quad \omega \equiv \omega_{1} \cdot e^{\lambda t} .
$$

Заметим, что исследование задачи (1.1)-(1.4) сводится к изучению задачи

ЗАДАЧА 2. Найти пару $u(x, t) \in W_{2,0}^{2,1}(Q), \varphi(x) \in L_{2}(\Omega)$ из условий

$$
\begin{aligned}
\rho u_{t}-L u+\lambda \rho u & =f(x, t) \equiv \Phi_{1}(x, t) e^{-\lambda t} p(x) \equiv \Phi(x, t) \varphi(x), & (x, t) \in Q,(1.12) \\
\left.u\right|_{t=0} & =0, & x \in \Omega,(1.13) \\
\left.u\right|_{S} & =0, & (x, t) \in S,(1.14) \\
l u & \equiv \int_{0}^{T} \omega(t) u(x, t) d t=\chi(x), & x \in \Omega,(1.15)
\end{aligned}
$$

с произвольной $\lambda \in \mathbb{R}$.

В самом деле, обозначив через $u_{2}$ решение прямой задачи (1.1)-(1.3) с $p(x) \equiv 0$, а за $\chi_{2}$ результат действия на него оператора переопределения:

$$
l_{1} u_{2}=\int_{0}^{T} \omega_{1}(t) u_{2}(x, t) d t=\chi_{2}(x)
$$

получим, что решения обратных задач $(1.1)-(1.4),(1.12)-(1.15)$ и указанной прямой задачи связаны соотношениями: $u_{1}=u \cdot e^{\lambda t}+u_{2}$, а $\chi_{1}=\chi+\chi_{2}$. При этом функции $u_{2}$ и $\chi_{2}$ известны; следовательно, решив (1.12)-(1.15), мы решим и (1.1)-(1.4). Осталось заметить, что при выполнении условий (1.9), (1.8) после перехода от задачи 1 к задаче 2 классы функций сохранятся. В частности, по лемме 1 функции $\chi, \chi_{1}$ и $\chi_{2}$ лежат в одном и том же пространстве $W_{2,0}^{2}(\Omega)$. Таким образом, имеет место взаимно-однозначное соответствие между множествами решений задач (1.12)-(1.15) и (1.1)-(1.4). Заметим, что в обозначениях (1.11) $l(\Phi(x)) \equiv 1$. Всюду далее будем исследовать задачу 2. Справедливы следуюшие утверждения: 
ТЕОрема 1. Пусть выполнены условия (1.6)-(1.11). Тогда задача (1.12)-(1.15) “фредгольмова", т.е. әквивалентна операторному уравнению Фредгольма 2-го рода с компактным оператором.

Чтобы сформулировать следующую теорему, введем обозначение: пусть функция $\alpha(t ; \lambda)$ такова, что при произвольной $\varphi \in L_{2}(\Omega)$ для решения $u(x, t)$ прямой задачи (1.13)-(1.15) с данной $f$ справедливо неравенство

$$
\left\|c_{t}(x, t) u+\lambda \rho_{t} u\right\|_{2, \Omega}^{2} \leqslant \alpha(t ; \lambda)\|f\|_{2, Q_{t}}^{2} .
$$

То, что такая функция существует, сразу следует из оценки $(2.16)[9$, гл. 3]: $\|u\| \leqslant$ $c\|f\|_{2, Q}$, и определения энергетической нормы $\|u\|$. Оценку для функции $\alpha(t ; \lambda)$ можно получить, например, при помощи леммы 2.

ТЕОрема 2. Пусть выполнены (1.6)-(1.11); функиия $\alpha(t ; \lambda)$ такова, что имеет место (1.16). Пусть также найдутся такие числа $\lambda u \varepsilon>0$, что имеют место неравенства

$$
\begin{gathered}
-b_{i x_{i}}+2 c+\lambda \rho+\rho_{t}-\frac{1}{\varepsilon} \geqslant 0 \\
m=2\|\omega\|_{2,(0, T)}^{2}\left(\rho_{1}+\frac{c_{\lambda}^{2} T^{2}}{2 \rho_{0}}\right) \int_{0}^{T} e^{-\lambda t}\left(\left\|\frac{\Phi^{2}(\cdot, 0)}{\rho(\cdot, 0)}\right\|_{\infty, \Omega}\right. \\
\left.+2 \varepsilon \int_{0}^{t} e^{\lambda \tau} \cdot\left(\left\|\Phi_{\tau}^{2}(\cdot, \tau)\right\|_{\infty, \Omega}+\tau \alpha(\tau ; \lambda)\left\|\Phi^{2}\right\|_{\infty, Q_{\tau}}\right) d \tau\right) d t<1
\end{gathered}
$$

где $c_{\lambda}=\max _{Q}|c(x, t)+\lambda \rho(x, t)|$. Тогда при любой $\chi(x) \in W_{2,0}^{2}(\Omega)$ существует единственное решение задачи (1.12)-(1.15), и имеет место оченка устойчивости:

$$
\|u\|_{2, Q}^{(2,1)}+\|\varphi\|_{2, \Omega} \leqslant c\|L \chi\|_{2, \Omega} .
$$

СЛЕДСТВИЕ 1. Пусть выполнены условия (1.6)-(1.11); функиия $\alpha(t ; \lambda)$ такова, что имеет место (1.16). Пусть также найдутся такие числа $\lambda u \varepsilon>0$, что имеют место неравенства (1.17) и

$$
\begin{gathered}
c(x, t)+\lambda \rho(x, t)=\tilde{c}(x) \geqslant 0 \\
m=\|\omega\|_{2,(0, T)}^{2} \rho_{1} \int_{0}^{T} e^{-\lambda t}\left(\left\|\frac{\Phi^{2}(\cdot, 0)}{\rho(\cdot, 0)}\right\|_{\infty, \Omega}\right. \\
\left.+2 \varepsilon \int_{0}^{t} e^{\lambda \tau}\left(\left\|\Phi_{\tau}^{2}(\cdot, \tau)\right\|_{\infty, \Omega}+\tau \alpha(\tau ; \lambda)\left\|\Phi^{2}\right\|_{\infty, Q_{\tau}}\right) d \tau\right) d t<1 .
\end{gathered}
$$

Тогда выполняются утверждения теоремы 2.

СЛЕДСТВИЕ 2. Пусть выполняется условие (1.5), а также условия теоремы 1 или следствия 1. Тогда при любой $\chi(x) \in W_{2,0}^{2}(\Omega)$ существует единственное решение задачи (1.1)-(1.4), и имеет место оченка устойчивости:

$$
\left\|u_{1}-u_{2}\right\|_{2, Q}^{(2,1)}+\|p\|_{2, \Omega} \leqslant c\|\chi\|_{2, \Omega}^{(2)}
$$

где $u_{2}$ - решение прямой задачи (1.1)-(1.3) при $p(x) \equiv 0$. 


\section{2. Существование и единственность решения задачи (1.12)-(1.15)}

2.1. Некоторые свойства решения прямой задачи. В этом пункте мы ради полноты изложения приведем лемму, содержащую необходимые при доказательстве теорем 1, 2 свойства повьшения гладкости решения прямой задачи (1.12)-(1.15) при повышении гладкости по $t$ правой части уравнения (1.12). Кроме того, получим оценку, аналогичную неравенству (6.3) работы [9, гл. 3$]$.

Будем обозначать через $u(x, t)$ решение прямой задачи $(1.12),(1.14)$ с начальным условием $u(x, 0)=u_{0}(x) \in W_{2,0}^{2}(\Omega)$ (на данную задачу мы будем ссылаться как на $(1.12),(1.2),(1.14))$. Для простоты положим в этом пункте $\lambda=0$. Функцию $f(x, t)$ в правой части (1.12) будем считать произвольной фиксированной функцией такой, что $f, f_{t} \in L_{2}(Q)$. Коэффициенты $a_{i j}, b_{i}, c$ оператора $L$ будем в данном пункте считать зависящими от $(x, t)$ функциями, обладающими следующей гладкостью:

$$
a_{i j} \equiv a_{j i}(x, t) \in C^{2}(\bar{Q}), \quad b_{i}(x, t) \in C^{1}(\bar{Q}), \quad c(x, t) \in C^{1}(\bar{Q}) .
$$

В силу большей гладкости по $t$ правой части уравнения (1.1) решение задачи (1.12), $(1.2),(1.14)$ обладает некоторьми дополнительньми дифференциальными свойствами. Для их формулировки рассмотрим вспомогательную прямую задачу: найти функиию $v(x, t) \in \stackrel{\circ}{V}_{2}^{1.0}(Q)$ из условий

$$
\begin{array}{lr}
\rho(x, t) v_{t}-L(x, t) v+\rho_{t}(x, t) v=F(x, t), & (x, t) \in Q, \\
\left.v\right|_{t=0}=\frac{1}{\rho(x, 0)}\left(f(x, 0)+L(x, 0) u_{0}(x)\right), & x \in \Omega, \\
\left.v\right|_{S}=0, & (x, t) \in S .
\end{array}
$$

Здесь $F(x, t)=f_{t}(x, t)+\left(a_{i j} u_{x_{i}}\right)_{x_{j}}-b_{i t} u_{x_{i}}-c_{t} u$, a $u(x, t)-$ решение $(1.12),(1.2),(1.14)$ с заданными $f$ из указанного класса и $u_{0}(x) \in W_{2,0}^{2}(\Omega)$. Поскольку $u(x, t)-$ сильное решение и имеет все производные, входящие в уравнение, суммируемые с квадратом, то $F(x, t) \in L_{2}(Q)$.

Решение $v \in \stackrel{\circ}{V}_{2}^{1,0}(Q)$ задачи (2.2)-(2.4) понимается в смысле выполнения интегрального тождества (см., например, [9])

$$
\begin{array}{r}
\int_{Q}\left(v \rho \mu_{t}-a_{i j} v_{x_{i}} \mu_{x_{j}}-b_{i} \mu v_{x_{i}}-c v \mu+F \mu\right) d x d t \\
+\int_{\Omega}\left(f(x, 0)+L(x, 0) u_{0}\right) \mu(x, 0) d x=0
\end{array}
$$

при всех $\mu \in W_{2,0}^{2,1}(Q)$ таких, что $\mu(x, T)=0$.

При вьполнении условий (1.6)-(1.8) и $f, f_{t} \in L_{2}(Q)$ задача (2.2)-(2.4) однозначно разрешима в $\stackrel{\circ}{V}_{2}^{1,0}(Q)$ (см. $[9$, гл. $\left.3, \S 4]\right)$.

Лемма 1. Пусть выполнены условия (1.6), (1.8), (2.1), $f, f_{t} \in L_{2}(Q), u_{0} \in W_{2,0}^{2}(\Omega)$. Тогда решение $v \in V_{2}^{1,0}(Q)$ задачи (2.2)-(2.4) и решение $u \in W_{2,0}^{2,1}(Q)$ прямой задачи (1.12), (1.2), (1.14) при $\lambda=0$ связаны соотношением $u_{t}=v$. При этом 
$v \in V_{2}^{1,0}(Q) \cap W_{2,0}^{2,1}\left(Q_{\varepsilon, T}\right)$, где $Q_{\varepsilon, T}=\Omega \times(\varepsilon, T)$ при каждом $\varepsilon \in(0, T)$, а при любом $t_{1} \in(0, T]$ существуют следы $u\left(x, t_{1}\right) \in W_{2,0}^{2}(\Omega), v\left(x, t_{1}\right) \in \stackrel{\circ}{W}_{2}^{1}(\Omega)$ и вернь оценки

$$
\begin{array}{r}
\|v\| \leqslant c_{1}\left(\|f\|_{2, Q}+\left\|f_{t}\right\|_{2, Q}+\|f(\cdot, 0)\|_{2, \Omega}+\left\|u_{0}\right\|_{2, \Omega}^{(2)}\right), \\
\left\|v\left(\cdot, t_{1}\right)\right\|_{2, \Omega}^{(1)} \leqslant c_{2}\left(\|f\|_{2, Q}+\left\|f_{t}\right\|_{2, Q}+\|f(\cdot, 0)\|_{2, \Omega}+\left\|u_{0}\right\|_{2, \Omega}^{(2)}\right),
\end{array}
$$

где $c_{1}=c_{1}(n, \rho, L), c_{2}=c_{2}(n, \rho, L, \varepsilon), t_{1} \in(\varepsilon, T]$.

Лемма 1 доказьвается аналогично подобным утверждениям работ [10]-[12], а также лемме 1.1.2 работы [1, гл. 1]. Подробное же ее доказательство для нашего случая приводится в совместной работе автора с проф. А. И. Прилепко, которая сдана в печать.

Теперь выведем оценку, аналогичную неравенству (6.3) работы [9, гл. 3].

Лемма 2. Пусть выполнены условия (1.6), (1.8), (2.1), $f, f_{t} \in L_{2}(Q), u_{0} \in W_{2,0}^{2}(\Omega)$. Тогда для решения $u(x, t)$ задачи (1.12), (1.2), (1.14) nри $u_{0} \in W_{2,0}^{2}(\Omega), \quad \lambda=0$ справедливы неравенства

$$
\begin{gathered}
2 \nu_{1}\left\|u_{x}\right\|_{2, Q_{t}}^{2}+\rho_{0}\|u(\cdot, t)\|_{2, \Omega}^{2} \leqslant\left(\frac{1}{\varepsilon_{0}}\|f\|_{2, Q_{t}}^{2}+\rho_{1}\left\|u_{0}\right\|_{2, \Omega}^{2}\right) \varkappa_{1}(t), \\
\text { əде } \varkappa_{1}(t)=1+\frac{1}{\rho_{0}} \int_{0}^{t} \mu_{3}(\tau) d \tau \exp \left(\frac{1}{\rho_{0}} \int_{0}^{t} \mu_{3}(\tau) d \tau\right) .
\end{gathered}
$$

Здесь $Q_{t}=\Omega \times(0, t), \varepsilon_{0}>0$ - любое положительное число. Остальные обозначения в (2.7)-(2.8) взяты аналогично [9]:

$$
\begin{gathered}
\mu_{2}(t)=\left|\max _{\Omega} \rho_{t}\right| \\
\mu_{3}(t) \geqslant 0 \quad \text { maкое, ито } \mu_{3}(t) \geqslant \mu_{2}(t)+\varepsilon_{0}+\max _{\Omega}\left(b_{i x_{i}}-2 c\right) .
\end{gathered}
$$

ДокАЗАТЕЛЬСтво. Для вьвода оценки (2.7) аналогично [9] рассмотрим тождество, которому должно удовлетворять решение $(1.12),(1.2),(1.14)$ в цилиндре $Q_{t}=\Omega \times(0, t)$ :

$$
\int_{Q_{t}}\left(\rho u_{\tau}-L u\right) u d x d \tau=\int_{Q_{t}} f u d x d \tau
$$

Так как $u(x, t)$ - сильное решение, оно удовлетворяет (1.13) п.в. в $Q$, и все используемые интегралы имеют смысл. Это тождество можно переписать в виде

$$
\int_{Q_{t}}\left(\frac{1}{2} \cdot\left(\rho u^{2}\right)_{\tau}-\frac{1}{2} \cdot \rho_{\tau} u^{2}+a_{i j} u_{x_{i}} u_{x_{j}}+b_{i} u_{x_{i}} u+c u^{2}\right) d x d \tau=\int_{Q_{t}} f u d x d \tau .
$$

Правую часть оценим по “неравенству Коши с эпсилон” [9, гл. $2, \S 1]$, а левую с использованием (1.8), (2.9) и граничных условий:

$$
\begin{aligned}
\left.\frac{1}{2} \int_{\Omega} \rho u^{2} d x\right|_{0} ^{t}-\frac{1}{2} \int_{0}^{t} \mu_{2}(\tau)\|u(\cdot, \tau)\|_{2, \Omega}^{2} d \tau+\nu_{1}\left\|u_{x}\right\|_{2, Q_{t}}^{2} \\
-\frac{1}{2} \int_{Q_{t}} b_{i x_{i}} u^{2} d x d \tau+\int_{Q_{t}} c u^{2} d x d \tau \leqslant \frac{1}{2 \varepsilon_{0}}\|f\|_{2, Q_{t}}^{2}+\frac{\varepsilon_{0}}{2}\|u\|_{2, Q_{t}}^{2}
\end{aligned}
$$


или, по-другому:

$$
\begin{aligned}
\int_{\Omega} \rho u^{2}(x, t) d x+2 \nu_{1}\left\|u_{x}\right\|_{2, Q_{t}}^{2} \leqslant & \int_{0}^{t} \mu_{2}(\tau)\|u(\cdot, \tau)\|_{2, \Omega}^{2} d \tau+\int_{Q_{t}}\left(b_{i x_{i}}-2 c\right) u^{2} d x d \tau \\
& +\int_{\Omega} \rho u_{0}^{2}(x) d x+\frac{1}{\varepsilon_{0}}\|f\|_{2, Q_{t}}^{2}+\varepsilon_{0}\|u\|_{2, Q_{t}}^{2} .
\end{aligned}
$$

Далее, с учетом (1.6) и (2.10)

$$
\rho_{0}\|u\|_{2, \Omega}^{2}+2 \nu_{1}\left\|u_{x}\right\|_{2, Q_{t}}^{2} \leqslant \int_{0}^{t} \mu_{3}(\tau)\|u(\cdot, \tau)\|_{2, \Omega}^{2} d \tau+\rho_{1}\left\|u_{0}\right\|_{2, \Omega}^{2}+\frac{1}{\varepsilon_{0}}\|f\|_{2, Q_{t}}^{2} .
$$

Введя функции

$$
y(t)=\rho_{0} \int_{0}^{t} \mu_{3}(\tau)\|u(\cdot, \tau)\|_{2, \Omega}^{2} d \tau \quad \text { и } \quad G(t)=\rho_{1}\left\|u_{0}\right\|_{2, \Omega}^{2}+\frac{1}{\varepsilon_{0}}\|f\|_{2, Q_{t}}^{2},
$$

из последнего неравенства для $y(t)$ получим

$$
y^{\prime}(t) \leqslant \frac{\mu_{3}(t)}{\rho_{0}} y(t)+\mu_{3}(t) G(t)
$$

откуда по лемме $5.5[9$, гл. $2, \S 5]$

$$
y(t) \leqslant \int_{0}^{t} \mu_{3}(\tau) G(\tau) d \tau \cdot \exp \left(\frac{1}{\rho_{0}} \int_{0}^{t} \mu_{3}(\tau) d \tau\right)
$$

а поскольку $G(t)$ монотонно неубьвающая неотрицательная функция, то

$$
y(t) \leqslant G(t) \int_{0}^{t} \mu_{3}(\tau) d \tau \cdot \exp \left(\frac{1}{\rho_{0}} \int_{0}^{t} \mu_{3}(\tau) d \tau\right) .
$$

Подставив эту оценку в неравенство (2.11), имеем (2.7). Лемма 2 доказана.

2.2. “Фредгольмовость” задачи (1.12)-(1.15). Обозначим через $L_{0}$ стационарную часть оператора $L$, т.е.

$$
L_{0} u=\left(a_{i j}(x) u_{x_{i}}\right)_{x_{j}}-b_{i}(x) u_{x_{i}}
$$

Вьполнение условий (1.6)-(1.9) гарантирует нам при каждом фиксированном $\varphi \in L_{2}(\Omega)$ существование единственного решения $u(x, t ; \varphi) \in W_{2,0}^{2,1}(Q)$ задачи $(1.12)-(1.14)[9$, гл. 3 , $\S 6 ;$ гл. $4, \S 9$ ]. Поэтому выражение

$$
A \varphi \equiv \int_{0}^{T} \omega(t) \rho(x, t) u_{t}(x, t ; \varphi) d t+\int_{0}^{T} \omega(t)(c(x, t)+\lambda \rho(x, t)) u(x, t ; \varphi) d t
$$

задает в силу леммы 1 линейньй оператор $A: L_{2}(\Omega) \rightarrow L_{2}(\Omega)$. Повьшение гладкости решения $u(x, t)$ (лемма 1 ) позволяет нам подействовать оператором переопределения $l$ на равенство (1.12). Заметим, что $l\left(L_{0} u\right)=L_{0}(l u)=L_{0} \chi$. Учитьвая, что в силу (1.11) $l(\Phi) \equiv 1$, а также определение (2.13) оператора $A$, получим из (1.12) следующее операторное уравнение второго рода:

$$
\begin{array}{cl}
A \varphi-\varphi=\psi, & \text { где } \psi=L_{0} \chi, \\
\text { при этом } & \left.\chi\right|_{\partial \Omega}=0 .
\end{array}
$$


ОпРЕДЕлЕниЕ. Будем говорить, что задача нахождения пары $u \in W_{2,0}^{2,1}(Q), \varphi \in$ $L_{2}(\Omega)$ из условий (1.12)-(1.15) әквивалентна задаче нахождения $\varphi \in L_{2}(\Omega)$ из $(2.14)$, (2.15) с оператором $A$, если при произвольной фиксированной $\chi(x) \in W_{2,0}^{2}(\Omega)$ вьполнены следующие условия:

1) если $(u, \varphi)$ - решение (1.12)-(1.15), то $\varphi$ - решение (2.14), (2.15);

$2)$ если $\varphi$ - решение $(2.14),(2.15)$, то пара $(u, \varphi),($ где $u(x, t)$ - решение $(1.12)-(1.14)$ с данной $\varphi$ ) является решением (1.12)-(1.15);

3) если не может быть двух разньх решений (1.12)-(1.15), то не может быть двух разных решений $(2.14),(2.15)$;

4) если не может быть двух разных решений (2.14), (2.15), то не может быть двух разных решений (1.12)-(1.15).

ДокАЗАТЕЛЬСТво ТЕОРЕмы 1 разбивается на два этапа: сначала докажем, что задача (1.12)-(1.15) эквивалентна задаче (2.14), (2.15), а затем убедимся, что оператор $A$, как оператор, действуюший из $L_{2}(\Omega)$ в $L_{2}(\Omega)$, является вполне непрерывным.

УТВЕРЖДЕНИЕ 1. Пусть выполнены условия (1.6)-(1.11). Тогда задача (1.12)(1.15) әквивалентна задаче (2.14), (2.15).

ДокАЗАТЕЛЬСтво. Вьполнение условия 1) следует из алгоритма вывода уравнений (2.14), (2.15).

Условие 2). Пусть $\varphi_{1} \in L_{2}(\Omega)$ есть решение $(2.14),(2.15)$ с заданной $\chi(x)$. Решим задачу (1.12)-(1.14) с $\varphi_{1}(x)$ вместо $\varphi(x)$ и обозначим решение через $u_{1}$. Повторим рассуждения по выводу $(2.14),(2.15)$ и получим

$$
A \varphi_{1}-\varphi_{1}=\int_{0}^{T} \omega L_{0} u_{1} d t=L_{0} \chi_{1}
$$

Но так как $\varphi_{1}$ - решение $(2.14),(2.15)$, то $L_{0} \chi_{1}=L_{0} \chi$, при этом $\chi_{1}, \chi \in W_{2,0}^{2}(\Omega)$. Отсюда в силу определения $L_{0}(2.12) \chi_{1} \equiv \chi$ и, наконец, пара $\left(u_{1}, \varphi_{1}\right)$ - решение $(1.12)-$ (1.15).

Условие 3). Пусть при некотором $\chi \in W_{2,0}^{2}(\Omega)$ найдется пара функций $\varphi_{1,2} \in L_{2}(\Omega)$ таких, что $A \varphi_{1,2}-\varphi_{1,2}=L \chi$. Обозначим решения задачи (1.12)-(1.14) с этими $\varphi_{1,2}$ через $u_{1,2}$ соответственно. Рассуждая, как при доказательстве условия 2), получим $l u_{1,2}=\chi$. Но тогда имеются две различные пары $\left(u_{1,2}, \varphi_{1,2}\right)$ решений задачи $(1.12)-$ (1.15), что противоречит предположению в условии 3$)$.

Условие 4). Пусть $\exists\left(u_{1,2}, \varphi_{1,2}\right)$ - пара решений (1.12)-(1.15). В силу вьполнения условия 1) $\varphi_{1,2}$ - пара решений $(2.14),(2.15)$. Полученное противоречие с предположением в условии 4) и завершает доказательство утверждения.

УТВЕРЖДЕНИЕ 2. Пусть выполнены условия (1.6)-(1.9). Тогда оператор А: $L_{2}(\Omega) \rightarrow L_{2}(\Omega)$ вполне непрерывен.

ДокАЗАтЕльство. Докажем сначала неравенство

$$
\|A \varphi\|_{2, \Omega}^{(1)} \leqslant c\|\varphi\|_{2, \Omega} .
$$


По лемме 1 функция $v=u_{t}$ является слабым решением следующей прямой задачи:

$$
\begin{cases}\rho(x, t) v_{t}-L(x, t) v+\left(\rho_{t}+\lambda \rho\right) v=F(x, t), & \left.v\right|_{S}=0 \\ \left.v\right|_{t=0}=v_{0} \equiv \frac{f(x, 0)}{\rho(x, 0)}, & x \in \Omega\end{cases}
$$

где $F(x, t)=f_{t}(x, t)-\left(c_{t}(x, t)+\lambda \rho_{t}(x, t)\right) u$. При этом $v\left(\cdot, t_{1}\right) \in \stackrel{\circ}{W}_{2}^{1}(\Omega)$ при произвольном $t_{1} \in(0, T]$, и вьполнены оценки $(2.5),(2.6)$.

Напомним, что оператор $A$ определен в $(2.13)$, где $u(x, t)=u(x, t ; \varphi)$ - сильное решение прямой задачи (1.12)-(1.14) при заданной $\varphi \in L_{2}(\Omega)$.

Чтобы получить оценку нормы $\|A \varphi\|_{2, \Omega}^{(1)}$, оценим сначала квадрат нормы первого слагаемого (2.13):

$$
\left(\left\|\int_{0}^{T} \omega(t) \rho(x, t) u_{t}(x, t ; \varphi) d t\right\|_{2, \Omega}^{(1)}\right)^{2} \equiv \int_{\Omega}\left(\left(l\left(\rho u_{t}\right)\right)^{2}+\left(l\left(\rho u_{t}\right)\right)_{x}^{2}\right) d x .
$$

По неравенству Коши-Буняковского получим

$$
\begin{aligned}
& \left(\left\|\int_{0}^{T} \omega(t) \rho(x, t) u_{t}(x, t ; \varphi) d t\right\|_{2, \Omega}^{(1)}\right)^{2} \\
& \quad \leqslant\|\omega\|_{2,(0, T)}^{2}\left(\int_{Q}\left(\left(\rho^{2}+\rho_{x}^{2}\right) u_{t}+\rho^{2}\left(u_{t}\right)_{x}^{2}\right) d x d t\right) \leqslant c\left(\left\|u_{t}\right\|_{2, Q}^{(1,0)}\right)^{2} .
\end{aligned}
$$

Далее, в силу оценки (2.5) леммы 1 при $u_{0}(x)=0$ имеем

$$
\left\|u_{t}\right\|_{2, Q}^{(1,0)} \leqslant\left\|u_{t}\right\| \leqslant c\left(\|f\|_{2, Q}+\|f(x, 0)\|_{2, \Omega}+\left\|f_{t}\right\|_{2, Q}\right) .
$$

Поскольку при $f(x, t)=\Phi(x, t) \varphi(x)$ очевидно неравенство

$$
\|f\|_{2, Q}+\left\|f_{t}\right\|_{2, Q}+\|f(x, 0)\|_{2, \Omega} \leqslant c\|\varphi\|_{2, \Omega}
$$

для первого слагаемого (2.13) имеем

$$
\left\|\int_{0}^{T} \omega(t) \rho(x, t) u_{t}(x, t ; \varphi) d t\right\|_{2, \Omega}^{(1)} \leqslant c\|\varphi\|_{2, \Omega} .
$$

Аналогично для нормы второго слагаемого в $(2.13)$ в силу гладкости $\rho(x, t)(1.6)$ и коэффициентов оператора $L(1.7)$, а также неравенства Коши-Буняковского, имеем

$$
\left\|\int_{0}^{T} \omega(t)(c(x, t)+\lambda \rho(x, t)) u(x, t ; \varphi) d t\right\|_{2, \Omega}^{(1)} \leqslant c\|u\|_{2, Q}^{(1,0)} .
$$

Заметим, что $\|u\|_{2, Q}^{(1,0)} \leqslant\|u\|_{2, Q}^{(2,1)}$, воспользуемся неравенством (9.3) работы [9, гл. 4]: $\|u\|_{2, Q}^{(2,1)} \leqslant c\|f\|_{2, Q}$ и (2.18). С учетом (2.19) получим неравенство $(2.16):\|A \varphi\|_{2, \Omega}^{(1)} \leqslant$ $c\|\varphi\|_{2, \Omega}$. Оно означает, что оператор $A$ переводит множество, ограниченное в норме $L_{2}(\Omega)$, во множество, ограниченное по норме $W_{2}^{1}(\Omega)$, которое компактно в $L_{2}(\Omega)$ по теореме Реллиха [13, гл. 1, теорема 6.1].

Таким образом, оператор $A$ компактен. Утверждение 2 , а вместе с ним и теорема 1 доказаны. 


\section{3. Существование и единственность "в малом".}

ДОКАЗАТЕЛЬСТВО ТЕОРЕМЫ 2. ЧтобЫ оценить норму

$$
\|A \varphi\|_{2, \Omega}^{2} \leqslant 2 \int_{\Omega}\left(\left(\int_{0}^{T} \omega \rho v d t\right)^{2}+\left(\int_{0}^{T} \omega(c+\lambda \rho) u d t\right)^{2}\right) d x
$$

сначала заметим, что

$$
\left(\int_{0}^{T} \omega \rho v d t\right)^{2}+\left(\int_{0}^{T} \omega c u d t\right)^{2} \leqslant\|\omega\|_{2,(0, T)}^{2}\left(\int_{0}^{T} \rho^{2} v^{2} d t+\int_{0}^{T}(c+\lambda \rho)^{2} u^{2} d t\right) .
$$

Далее, поскольку

$$
\begin{aligned}
\|u\|_{2, Q}^{2} & =\int_{Q}\left(\int_{0}^{t} 1 \cdot u_{\tau} d \tau\right)^{2} d x d t \leqslant \int_{Q} t \cdot \int_{0}^{t} u_{\tau}^{2} d \tau d x d t \\
& \leqslant \int_{0}^{T} t \cdot\left\|u_{t}\right\|_{2, Q}^{2} d t=\frac{T^{2}}{2} \cdot\left\|u_{t}\right\|_{2, Q}^{2},
\end{aligned}
$$

и

$$
\int_{Q}(c+\lambda \rho)^{2} u^{2} d x d t \leqslant c_{\lambda}^{2} \frac{T^{2}}{2} \int_{Q} v^{2} d x d t \leqslant \frac{c_{\lambda}^{2} T^{2}}{2 \rho_{0}} \int_{Q} \rho v^{2} d x d t,
$$

для нормы $\|A \varphi\|_{2, \Omega}^{2}$ имеем

$$
\|A \varphi\|_{2, \Omega}^{2} \leqslant 2\|\omega\|_{2,(0, T)}^{2} \cdot\left(\rho_{1}+\frac{c_{\lambda}^{2} T^{2}}{2 \rho_{0}}\right) \cdot \int_{Q} \rho v^{2} d x d t .
$$

Осталось оценить $\int_{Q} \rho v^{2} d x d t$. Пусть $\left\{f_{k}(x, t)\right\} \in C^{\infty}(\bar{Q})$ - последовательность функций, сходяшаяся в $L_{2}(Q)$ к $f(x, t)$ при $k \rightarrow \infty$, такая, что $\left.f_{k}(x, 0)\right|_{\text {ә }}=0, k=1, \ldots, \infty$. Обозначим через $u_{k}$ решения задач

$$
\begin{cases}\rho u_{k t}-L u_{k}+\lambda \rho u_{k}=f_{k}(x, t), & (x, t) \in Q, \\ \left.u_{k}\right|_{t=0}=0, & x \in \Omega, \\ \left.u_{k}\right|_{S}=0, & (x, t) \in S .\end{cases}
$$

Тогда по лемме 1 функции $v_{k} \equiv u_{k}$ являются решениями задач

$$
\begin{cases}\rho v_{k t}-L v_{k}+\left(\lambda \rho+\rho_{t}\right) v_{k}=F_{k}(x, t) \equiv f_{k t}-\left(c_{t}+\lambda \rho_{t}\right) u_{k}, & (x, t) \in Q, \\ \left.v_{k}\right|_{t=0}=\frac{f_{k}(x, 0)}{\rho(x, 0)}, & x \in \Omega, \\ \left.v_{k}\right|_{S}=0, & (x, t) \in S .\end{cases}
$$

В силу выбора последовательности $f_{k}$ решения задач $(2.22),(2.23)$ - сильные (и даже классические $[14$, гл. $3, \S 4])$, поэтому имеет смысл следующая операция: домножим уравнение в $(2.23)$ на $v_{k}(x, t)$ и проинтегрируем по $Q_{t}=\Omega \times(0, t)$. Получим

$$
\int_{Q_{t}}\left(\rho v_{k t}-L v_{k}+\left(\lambda \rho+\rho_{t}\right) v_{k}\right) v_{k} d x d t=\int_{Q_{t}} F_{k}(x, t) v_{k}(x, t) d x d t .
$$


Так как $2 \rho v_{k} v_{k}=\left(\rho v_{k}^{2}\right)_{t}-\rho_{t} v_{k}^{2}$, а $2 \int_{\Omega} b_{i} v_{k x_{i}} v_{k} d x=-\int_{\Omega} b_{i} x_{i} v_{k}^{2} d x$ при $\left.v_{k}\right|_{\partial \Omega}=0$, продифференцировав данное равенство по $t$, получим

$$
\left(\int_{\Omega} \rho v_{k}^{2} d x\right)_{t}+\int_{\Omega}\left(2 a_{i j} v_{k x_{i}} v_{k x_{j}}+\left(-b_{i x_{i}}+2 c+\rho_{t}+2 \lambda \rho\right) v_{k}^{2}\right) d x=2 \int_{\Omega} F_{k} v_{k} d x .
$$

Пренебрежем неотрицательным слагаемым $a_{i j} v_{k} x_{i} v_{k} x_{j} \geqslant \nu_{1} v_{k x}^{2} \geqslant 0$ в левой части, а правую оценим по неравенству Коши с эпсилон:

$$
\left(\int_{\Omega} \rho v_{k}^{2} d x\right)_{t}+\int_{\Omega}\left(-b_{i x_{i}}+2 c+\rho_{t}+2 \lambda \rho\right) v_{k}^{2} d x \leqslant \varepsilon\left\|F_{k}(\cdot, t)\right\|_{2, \Omega}^{2}+\frac{1}{\varepsilon}\left\|v_{k}(\cdot, t)\right\|_{2, \Omega}^{2},
$$

или

$$
\left(\int_{\Omega} \rho v_{k}^{2} d x\right)_{t}+\int_{\Omega}\left(-b_{i x_{i}}+2 c+\rho_{t}+\lambda \rho-\frac{1}{\varepsilon}\right) v_{k}^{2} d x \leqslant-\lambda \int_{\Omega} \rho v_{k}^{2} d x+\varepsilon\left\|F_{k}(\cdot, t)\right\|_{2, \Omega}^{2} .
$$

В силу произвольности $\lambda>0$ и условий $(1.6),(1.7) \lambda$ может быть выбрано настолько большим, чтобы вьполнялось (1.17). При таком выборе $\lambda$ имеем неравенство

$$
\left(\int_{\Omega} \rho v_{k}^{2} d x\right)_{t} \leqslant-\lambda \int_{\Omega} \rho v_{k}^{2} d x+\varepsilon\left\|F_{k}(\cdot, t)\right\|_{2, \Omega}^{2}
$$

Обозначив $y(t)=\int_{\Omega} \rho v_{k}^{2} d x$, для функции $y(t)$ имеем неравенство

$$
y^{\prime}(t) \leqslant-\lambda y(t)+\varepsilon\left\|F_{k}(\cdot, t)\right\|_{2, \Omega}^{2} \quad \text { или } \quad\left(y(t) e^{\lambda t}\right)_{t} \leqslant e^{\lambda t} \varepsilon\left\|F_{k}(\cdot, t)\right\|_{2, \Omega}^{2} .
$$

Проинтегрируем это неравенство от 0 до $t$ и учтем начальные данные:

$$
y(t) \leqslant\left(\int_{\Omega} \frac{f_{k}^{2}(x, 0)}{\rho(x, 0)} d x+\varepsilon \int_{0}^{t} e^{\lambda \tau}\left\|F_{k}(\cdot, \tau)\right\|_{2, \Omega}^{2} d \tau\right) \cdot e^{-\lambda t} .
$$

Перейдем теперь к пределу при $k \rightarrow \infty$ и используем определение функции $y(t)$ :

$$
\int_{\Omega} \rho(x, t) v^{2}(x, t) d x \leqslant\left(\int_{\Omega} \frac{f^{2}(x, 0)}{\rho(x, 0)} d x+\varepsilon \int_{0}^{t} e^{\lambda \tau}\|F(\cdot, \tau)\|_{2, \Omega}^{2} d \tau\right) \cdot e^{-\lambda t} .
$$

Учтем теперь вид функции $F \equiv f_{t}-c_{t} u-\lambda \rho_{t} u$ и условие (1.16). Получим

$$
\begin{aligned}
\int_{0}^{t} e^{\lambda \tau}\|F(\cdot, \tau)\|_{2, \Omega}^{2} d \tau & \leqslant 2 \int_{0}^{T} e^{\lambda \tau}\left(\left\|f_{\tau}(\cdot, \tau)\right\|_{2, \Omega}^{2}+\alpha(\tau ; \lambda)\|f\|_{2, Q_{\tau}}^{2}\right) d t \\
& \leqslant 2 \int_{0}^{t} e^{\lambda \tau}\left(\left\|\Phi_{\tau}^{2}(\cdot, \tau)\right\|_{\infty, \Omega}+\alpha(\tau ; \lambda)\left\|\Phi^{2}\right\|_{\infty}, Q_{\tau} \cdot \tau\right) d \tau \cdot\|\varphi\|_{2, \Omega}^{2} .
\end{aligned}
$$


Подставим в $(2,24)$ :

$$
\begin{aligned}
\int_{\Omega} \rho(x, t) v^{2}(x, t) d x \leqslant & e^{-\lambda t} \cdot\left(\left\|\frac{\Phi^{2}(\cdot, 0)}{\rho(\cdot, 0)}\right\|_{\infty, \Omega}\right. \\
& \left.+2 \varepsilon \int_{0}^{t} e^{\lambda \tau}\left(\left\|\Phi_{t}^{2}(\cdot, \tau)\right\|_{\infty, \Omega}+\alpha(\tau ; \lambda)\left\|\Phi^{2}\right\|_{\infty, Q_{\tau}} \cdot \tau\right) d \tau\right) \cdot\|\varphi\|_{2, \Omega}^{2} .
\end{aligned}
$$

Наконец, оценим норму оператора $A$. В силу (2.21) имеем

$$
\begin{aligned}
\|A \varphi\|_{2, \Omega}^{2} \leqslant & 2\|\omega\|_{2,(0, T)}^{2} \cdot\left(\rho_{1}+\frac{c_{\lambda}^{2} T^{2}}{2 \rho_{0}}\right) \int_{0}^{T} e^{-\lambda t}\left(\left\|\frac{\Phi^{2}(\cdot, 0)}{\rho(\cdot, 0)}\right\|_{\infty, \Omega}\right. \\
& \left.+2 \varepsilon \int_{0}^{t} e^{\lambda \tau}\left(\left\|\Phi_{t}^{2}(\cdot, \tau)\right\|_{\infty, \Omega}+\alpha(\tau ; \lambda)\left\|\Phi^{2}\right\|_{\infty, Q_{\tau}} \cdot \tau\right) d \tau\right) d t \cdot\|\varphi\|_{2, \Omega}^{2} .
\end{aligned}
$$

Поэтому, если имеет место (1.18), то вьполняется неравенство $\|A\| \leqslant \sqrt{m}<1$. Таким образом, оператор $A$ является сжимающим; следовательно, $A-I$ обратим, и при каждом $\chi \in W_{2,0}^{2}(\Omega)$ задача $(2.14),(2.15)$ имеет единственное решение $\varphi(x) \in L_{2}(\Omega)$. Следовательно, по утверждению 1 имеет единственное решение $(u, \varphi)$ задача $(1.12)-(1.15)$.

При этом, так как $\|\varphi\|_{2, \Omega} \leqslant\|A \varphi\|_{2, \Omega}+\|L \chi\|_{2, \Omega}$, то $\|\varphi\|_{2, \Omega} \leqslant\|L \chi\|_{2, \Omega} /(1-\sqrt{m})$, а поскольку $\|u\|_{2, Q}^{(2,1)} \leqslant c\|\varphi\|_{2, \Omega}$ в силу [9, гл. 4, § 9], для решения (1.12)-(1.15) имеем оценку устойчивости:

$$
\|u\|_{2, Q}^{(2,1)}+\|\varphi\|_{2, \Omega} \leqslant c\|L \chi\|_{2, \Omega} .
$$

Теорема 2 доказана.

ДОКАЗАТЕЛЬСТВО С ЛЕДСТВИЯ 1. В данном, болеепростом, случае обратную задачу (1.12)-(1.15) удобнее свести к эквивалентному операторному уравнению с несколько отличным оператором:

$$
A_{1} \varphi=\int_{0}^{T} \omega(t) \rho(x, t) u_{t}(x, t ; \varphi) d t .
$$

Эквивалентность (1.12)-(1.15) и уравнения

$$
\begin{gathered}
A_{1} \varphi-\varphi=\psi, \\
\text { где } \quad \psi=(L-\lambda \rho)(x) \chi,
\end{gathered}
$$

доказьвается полностью аналогично утверждению 1, учитьвая те факты, что, во-первых, оператор $L-\lambda \rho$ является стационарным и, следовательно, перестановочным с оператором переопределения $l$ и, во-вторых, что при выполнении неравенства (1.19) задача $(L-\lambda \rho) \chi=\psi,\left.\chi\right|_{\partial \Omega}=0$ будет однозначно разрешима при любой $\psi \in L_{2}(\Omega)$.

Норма $\left\|A_{1} \varphi\right\|_{2, \Omega}^{(1)}$ оценивается в точности так же, как норма первого слагаемого при доказательстве утверждения 2 .

Таким образом, задача (1.12)-(1.15) в частном случае, соответствующем условиям следствия 1 , сводится к (2.26)-(2.27). Поэтому, если будет доказано неравенство $\left\|A_{1}\right\|<1$ при выполнении условий следствия 1 , то мы сразу получаем все утверждения теоремы для этого случая. 
Сопоставляя (2.25) и (2.13), видим, что норма $\left\|A_{1} \varphi\right\|_{2, \Omega}^{2}$ оценивается через норму первого слагаемого в выражении (2.13), определяющем оператор $A$, откуда вместо (2.21) имеем

$$
\left\|A_{1} \varphi\right\|_{2, \Omega}^{2} \leqslant \rho_{1}\|\omega\|_{2,(0, T)}^{2} \cdot \int_{Q} \rho v^{2} d x d t .
$$

Оценка $\int_{Q} \rho v^{2} d x d t$ проводится так же, как в доказательстве теоремы 2 . В результате мы получаем оценку (1.20) вместо (1.18). Следствие 1 доказано.

ДОКАЗАТЕЛЬСТВО СЛЕДСТВИя 2 сводится к повторению рассуждений п. 1.2 о сведении задачи (1.1)-(1.4) к задаче (1.12)-(1.15) и применению к (1.12)-(1.15) теоремы 2 и очевидного в силу (1.7) неравенства $\|L \chi\|_{2, \Omega} \leqslant c\|\chi\|_{2, \Omega}^{(2)}$.

\section{4. Примеры использования теоремы 2 .}

ПРИМЕР 1. Пусть $L u=\left(a_{i j}(x) u_{x_{i}}\right)_{x_{j}}-b_{i} u_{x_{i}}$ при $b_{i}=$ const, a $\rho=\rho(x)$. Тогда $L_{t} u \equiv 0, \rho_{t} \equiv 0$ и, следовательно, $\alpha(t ; \lambda) \equiv 0$. Пусть $\omega(t) \equiv 1$ и $\Phi_{1}(x, t)=\Phi_{2}(x) e^{\delta t}$ при некотором $\delta>0$. При таких условиях, выбрав сразу $\lambda=\delta$, мы получим

$$
\Phi=1 / \int_{0}^{T} \omega(t) d t=\frac{1}{T}
$$

и (1.20) перепишется в виде

$$
m=\rho_{1} \cdot T^{2} \int_{0}^{T} e^{-\delta t}\left(\frac{1}{T^{2} \rho_{0}}+2 \varepsilon \cdot 0\right) d t<1 .
$$

Выбрав $\varepsilon$ произвольньм образом так, чтобы вьполнялось условие (1.17), и вычислив интеграл, получим

$$
m=\frac{\rho_{1}}{\rho_{0}} \cdot \int_{0}^{T} e^{-\delta t} d t=\frac{\rho_{1}}{\rho_{0}} \cdot \frac{1-e^{-\delta T}}{\delta}<1 .
$$

Отсюда имеем, что

1) при $\delta \geqslant \rho_{1} / \rho_{0}$ неравенство $m<1$ выполнено при любых $T>0$ и по следствию 1 задача (1.12)-(1.15) является корректно разрешимой при всех $T>0$;

$2)$ при $\delta<\rho_{1} / \rho_{0}$ неравенство (1.20) будет верно при достаточно малых $T$, а именно, при $T<\frac{1}{\delta} \ln \left(\rho_{1} /\left(\rho_{1}-\delta \rho_{0}\right)\right)$, и тогда же будет корректно разрешима задача (1.12)-(1.15).

ПримеР 2. Пусть, как и в примере $1, \Phi_{1}(x, t)=\Phi_{2}(x) e^{\delta t}$ при некотором $\delta>0$. При таких условиях, выбрав сразу $\lambda=\delta$, мы получим $\Phi=1 / T$. Пусть также выполняются все остальные условия примера 1 , но $c=c(x, t)$. Тогда число $\varepsilon$ можно подобрать из условия (1.17) по данным $c, \rho$ и $\lambda=\delta$. Функцию $\alpha(t ; \lambda) \not \equiv 0$ можно найти из неравенства

$$
\left\|L_{t} u\right\|_{2, \Omega}^{2} \leqslant \max _{\Omega}\left(c_{t}^{2}(x, t)\right) \cdot\|u\|_{2, \Omega}^{2}
$$

и оценки $(2.7)$ :

$$
\|u\|_{2, \Omega}^{2} \leqslant \frac{\varkappa(t)}{\rho_{0} \varepsilon_{0}}\|f\|_{2, Q_{t}}^{2},
$$


где $\varepsilon_{0}-$ любое положительное число, а

$$
\varkappa(t)=1+\frac{1}{\rho_{0}} \int_{0}^{t} \max _{\Omega}\left\{0, \varepsilon_{0}-2 c-2 \lambda \rho\right\} d \tau \cdot e^{\frac{1}{\rho_{0}} \int_{0}^{t} \max _{\Omega}\left\{0, \varepsilon_{0}-2 c-2 \lambda \rho\right\} d \tau} .
$$

Заметим, что вместо $c(x, t)$ в данной оценке мы подставили $c(x, t)+\lambda \rho$, в соответствии с видом левой части уравнения в задаче (1.12).

Если $c(x, t) \geqslant 0$, то $\varepsilon_{0}$ можно выбрать так, чтобы $\varkappa(t) \equiv 1$, положив $\varepsilon_{0}=\min _{Q}(2 c+$ $2 \lambda \rho)$. Обозначим

$$
\tilde{\alpha}=\max _{Q}\left(c_{t}^{2}(x, t)\right) \cdot \frac{1}{\varepsilon_{0}} .
$$

Тогда $\alpha(t ; \lambda) \leqslant \tilde{\alpha} / \rho_{0}$ и $(1.18)$ приобретет вид

$$
m \leqslant 2 T^{2}\left(\rho_{1}+\frac{c_{\delta}^{2} T^{2}}{2 \rho_{0}}\right) \cdot \int_{0}^{T} e^{-\delta t}\left(\frac{1}{T^{2} \rho_{0}}+2 \varepsilon \int_{0}^{t} e^{\delta \tau} \tau \cdot \frac{\tilde{\alpha}}{T^{2} \rho_{0}} d \tau\right) d t .
$$

Отсюда

$$
m \leqslant \frac{2}{\rho_{0}}\left(\rho_{1}+\frac{c_{\delta}^{2} T^{2}}{2 \rho_{0}}\right) \cdot\left[\frac{1-e^{-\delta T}}{\delta}+\frac{2 \tilde{\alpha} \varepsilon}{\delta^{2}}\left(\frac{\delta T^{2}}{2}-T+\frac{1-e^{-\delta T}}{\delta}\right)\right] .
$$

Таким образом, $m=O(T)$ при $T \rightarrow+0$, поэтому при $c(x, t) \geqslant 0$ и достаточно малых $T$ неравенство $m<1$ будет вьполнено, и обратная задача (1.12)-(1.15) будет корректно разрешима.

ЗАмЕчАниЕ 2.1. 1) Оценка (1.20) дает для случая $\rho(x, t) \equiv 1, c(x, t) \equiv c(x)$ результат, близкий к установленному в [2].

2) Теоремы 1,2 , а также следствия 1,2 можно обобщить на случай, когда требования на гладкость коэффициентов $\rho(x, t), a_{i j}(x), b_{i}(x), c(x, t)$ являются минимальными, необходимьми для корректности прямой задачи (1.12)-(1.14).

3) Теорему 2 и следствия 1 и 2 можно эквивалентно сформулировать следующим способом: поскольку $\varepsilon$ и $\lambda$ в определениях (1.18) и (1.20) числа $m$ могут быть выбраны произвольно, при единственном условии (1.17) (и (1.19) в случае следствия 1$)$, то $m$ в (1.18) и (1.20), фактически, является функцией от $\varepsilon$ и $\lambda: m=m(\varepsilon, \lambda)$. Поэтому вместо неравенства $m<1$ можно требовать выполнения условия

$$
\widetilde{m} \equiv \inf _{\varepsilon, \lambda}\{m(\varepsilon, \lambda)\}<1,
$$

где inf берется по всем $\varepsilon$ и $\lambda$, удовлетворяющим условию (1.17) (а для следствия 1 еще и (1.19)). Заметим, что в примерах 1 и 2 мы выбирали $\lambda$ не из соображений оптимальности, а для упрощения вычислений.

\section{СПИСОК ЦИТИРОВАННОЙ ЛИТЕРАТУРЫ}

[1] Prilepko A. I., Orlovsky D. G., Vasin I. A. Methods for Solving Inverse Problems in Mathematical Physics. New York-Basel: Marcel Dekker inc., 2000.

[2] Прилепко А. И., Костин А. Б. О некоторых обратных задачах для параболических уравнений с финальным и интегральным наблюдением // Матем. сб. 1992. Т. 183. № 4 . C. $49-68$.

[3] Прилепко А. И., Соловьев В. В. Теоремы разрешимости и метод Ротэ в обратных задачах для уравнений параболического типа // Дифференц. уравнения. 1987. Т. 23. № 11. C. 1971-1980. 
[4] Прилепко А. И., Тихонов И. В.Единственность решения обратной задачи для эволюционного уравнения и приложения к уравнению переноса // Матем. заметки. 1992. Т. 51. № 2. C. $77-87$.

[5] Прилепко А. И., Костин А. Б. Оценка спектрального радиуса одного оператора и разрешимость обратных задач для эволюционного уравнения // Матем. заметки. 1993. Т. 53. № 1. C. 89-94.

[6] Прилепко А. И., Васин И. А. Некоторые обратные начально-краевые задачи для нестационарных линеаризованных уравнений Навье-Стокса // Дифференц. уравнения. 1989. T. 25. № 1. C. $106-117$.

[7] Прилепко А. И., Орловский Д. Г. О полугрупповом подходе к задаче определения неоднородного члена в эволюционных уравнениях // Докл. АН СССР. 1989. Т. 305. № 5 . C. $1045-1049$.

[8] Isakov V. Inverse parabolic problems with the final overdetermination // Commun. on Pure and Appl. Math. 1991. V. 44. P. 185-209.

[9] Ладыженская О. А., Солонников В. А., Уральцева Н. Н. Линейные и квазилинейные уравнения параболического типа. М.: Наука, 1967.

[10] Ладыженская О. А. О решении нестационарных операторных уравнений // Матем. сб. 1956. Т. 39 (81). № 4. С. 491-524.

[11] Вишик М. И., Ладыженская О. А. Краевые задачи для уравнений в частных производных и некоторых классов операторных уравнений // УМН. 1956. Т. 11. № 6(72). С. 41-97.

[12] Ладыженская О. А. Математические вопросы динамики вязкой несжимаемой жидкости. 2-е издание. М.: Наука, 1970.

[13] Ладыженская О. А. Краевые задачи математической физики. М.: Наука, 1973.

[14] Фридман А. Уравнения с частньми производньми параболического типа. М.: Мир, 1968.

Московский инженерно-фризический институт

Поступило

E-mail: sdtk@mail1.lebedev.ru

06.06 .2002 